\title{
Preliminary design of a G-band extended interaction oscillator driven by a sheet electron beam
}

\author{
${ }^{1,2}$ Guoxiang Shu, ${ }^{1}$ Wenlong He, ${ }^{1}$ Liang Zhang, ${ }^{1}$ Huabi Yin, ${ }^{1}$ Adrian W. Cross, and ${ }^{1}$ Alan D.R. Phelps \\ ${ }^{1}$ Department of Physics, SUPA, University of Strathclyde, Glasgow, Scotland, UK, G4 0NG, \\ ${ }^{2}$ School of Physical Electronics, University of Electronic Science and Technology of China, Chengdu, China, 610054 \\ guoxiang.shu@strath.ac.uk
}

Keywords: extended interaction oscillator, sheet electron beam, sub-terahertz

\begin{abstract}
A preliminary design of a G-band extended interaction oscillator (EIO) driven by a sheet electron beam is presented in this paper. PIC-3D simulations reveal that an output power of about $3.1 \mathrm{~kW}$ can be achieved when driven by a sheet electron beam with a voltage of $31.5 \mathrm{kV}$ and a current of 0.85 A. The oscillation frequency is $197.3 \mathrm{GHz}$ and the electronic efficiency is about $11.6 \%$.
\end{abstract}

\section{Introduction}

In the millimeter and sub-millimeter wavelength community, RF sources with compact configuration, high efficiency, and high output power have many potential applications, including satellite communications, radars, molecular spectroscopy, bioimaging, and plasma science [1-11]. The extended interaction oscillator (EIO) has been widely studied as a promising source to satisfy some of these applications, due to its high radiation power and compact configuration [1-2]. Compared with pencil electron beam vacuum electron devices (VEDs), sheet beam devices have attracted considerable attention as they produce radiation power proportional to the beam width and reduce the electron beam current density, leading to low magnetic field strengths required for beam focusing, thereby enabling periodic-permanent-magnet (PPM) focusing [5-7].

A W-band EIO with a pseudospark-sourced [8-11] pencil electron beam was previously studied at the University of Strathclyde [2]. These experiments achieved a radiation power of about $38 \mathrm{~W}$ at $92 \mathrm{GHz}$. However, the output power will be subject to a large reduction with an increase in the operating frequency. Thus, to increase the radiation power in the terahertz region, the pencil electron beam is replaced by a sheet electron beam to drive the G-band EIO. Output power can be greatly increased by enlarging the sheet beam cross section.

\section{Preliminary design}

A preliminary design of a G-band sheet beam EIO is presented in this paper, including the cold cavity analysis and PIC-3D numerical calculation. As shown in Fig. 1, the beam wave interaction structure of the EIO consists of a beam tunnel, two coupling cavities, a waveguide aperture, an output standard waveguide, and 11 gaps. Its structural dimensions are optimized to achieve an electric field (E-field) with good uniformity across the beam width. In addition, the characteristic impedance $(\mathrm{R} / \mathrm{Q})$ is expected to become as large as can be supported by the structure. The EIO operates in the $2 \pi \mathrm{TM}_{01}$-like mode and its E-field distribution is given in Fig. 1. As depicted in Fig. 2, the R/Q for the interaction circuit reached a high level of 835 at the operating mode. The potential mode competition can be suppressed due to the very small R/Q for other adjacent modes.

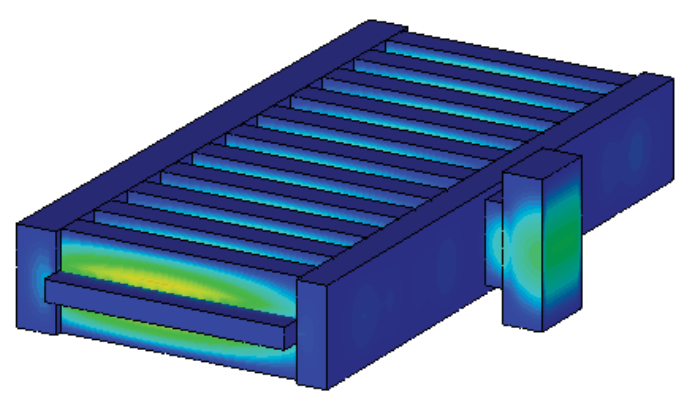

Fig. 1 Schematic of the designed EIO and its E-field distribution.

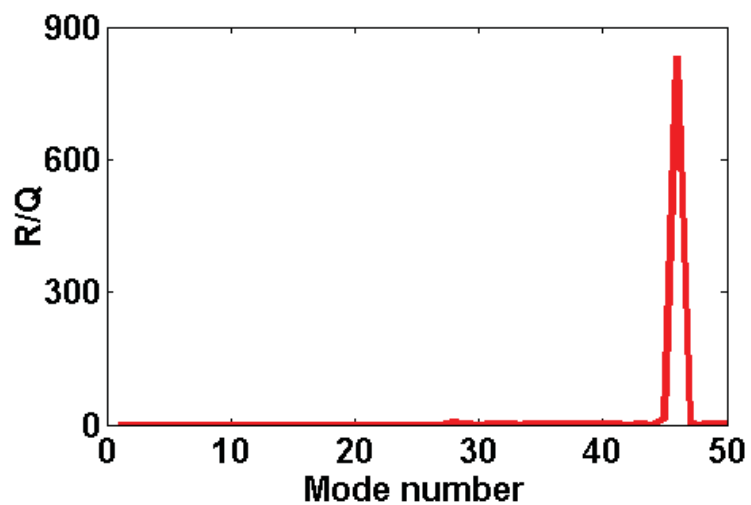

Fig. 2 The R/Q for different modes.

CST-PS is employed to study the beam wave interaction for the EIO. The RF circuit is driven by a high aspect ratio (13.6:1) sheet beam with a voltage of $31.5 \mathrm{kV}$ and a current of $0.85 \mathrm{~A}$. As depicted in Fig. 3, most of the electrons have converted their kinetic energies to the electromagnetic wave at the output port. A stable output is observed at the output port, as given in Fig. 4. It is demonstrated that the beam wave interaction has 
produced a radiation power of $3.1 \mathrm{~kW}$, and the corresponding electronic efficiency is about $11.6 \%$.

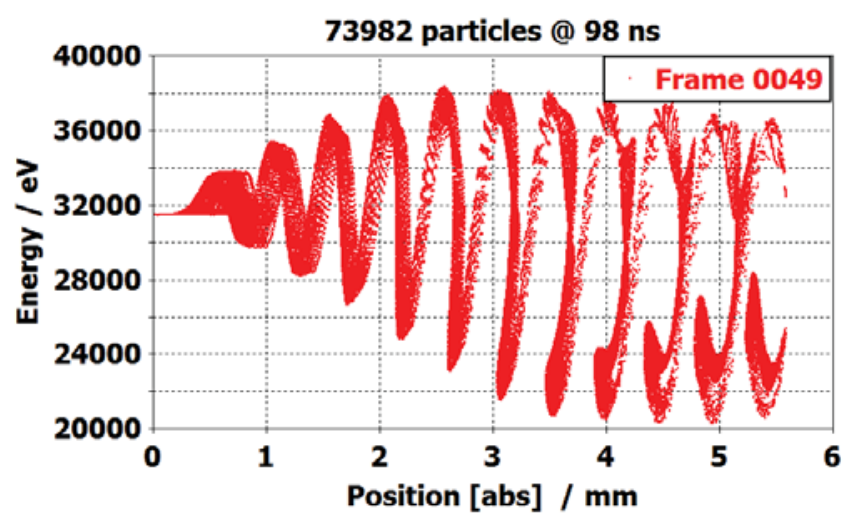

Fig. 3 The PIC phase space monitored at 98ns.

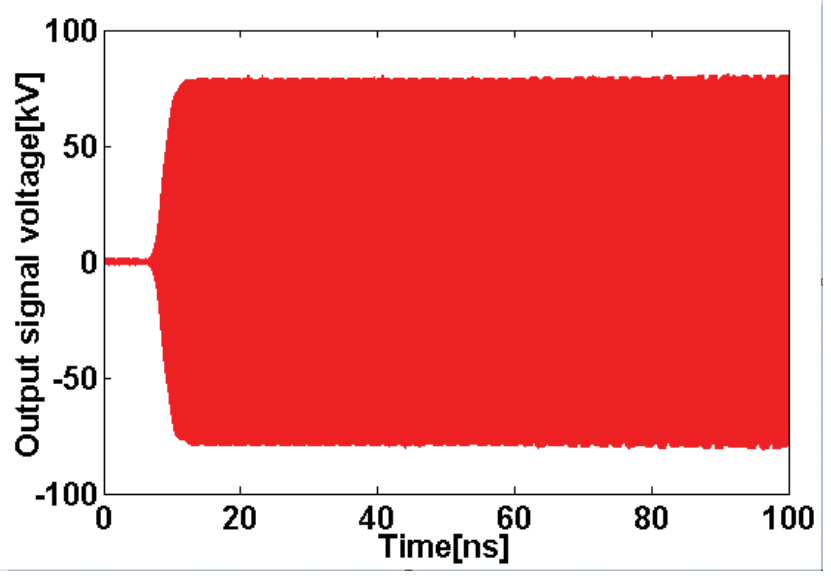

Fig. 4 The magnitude of the time-domain output signal.

As shown in Fig. 5, the output signal has a pure frequency spectrum. The frequency spectrum only peaks at $197.3 \mathrm{GHz}$, which is in good agreement with the cold cavity analysis. PIC simulations were also conducted using MAGIC-3D, and the simulation results from these two simulation tools are in good agreement.

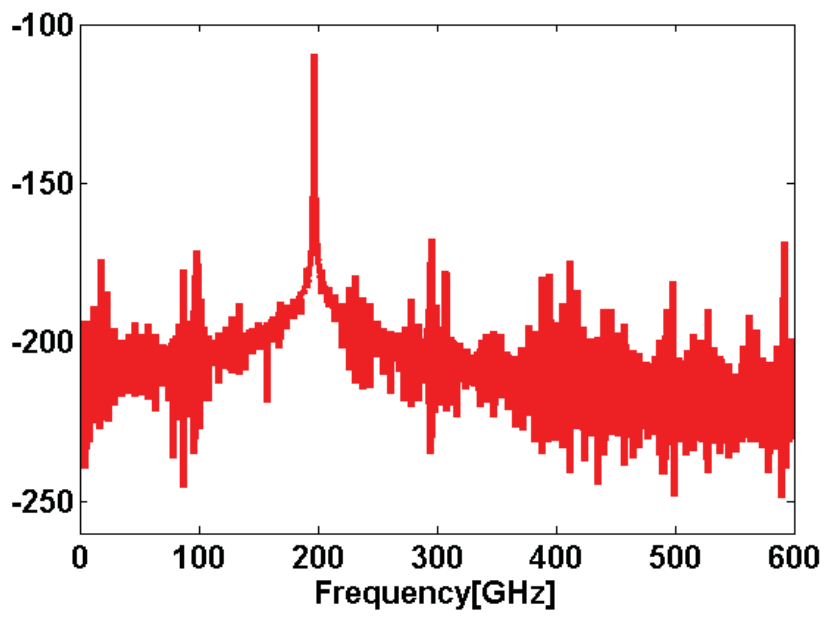

Fig. 5 The frequency spectrum of the output signal.

\section{Conclusion}

A sheet beam EIO has been preliminary designed to produce a radiation power of about $3.1 \mathrm{~kW}$ at around $200 \mathrm{GHz}$, which can act as a sub-terahertz radiation source with high power. A pseudo-spark discharge system will be employed to drive the beam wave interaction circuit, which is now under construction. A G-band EIO based on the pseudospark-sourced sheet electron beam is planned to be experimentally tested at the University of Strathclyde in the near future.

\section{Acknowledgement}

The authors would like to thank the Engineering and Physical Sciences Research Council (EPSRC) for supporting this work.

\section{References}

[1] Y. Yin, W. He, L. Zhang, H. Yin, and A. W. Cross, "Preliminary design and optimization of a G-band extended interaction oscillator based on a pseudosparksourced electron beam," Phys. Plasmas, vol. 22, no. 7, pp. 073102-1-073102-6, Jul. 2015.

[2] Yong Yin, Wenlong He, Liang Zhang, Huabi Yin, Craig W. Robertson and Adrian W. Cross, "Simulation and experiments of a W-band Extended Interaction Oscillator based on a pseudospark-sourced electron beam," IEEE Trans. Electron Devices, vol. 63, no. 1, pp. 512-516, Jan. 2016.

[3] W. He, L. Zhang, D. Bowes, H. Yin, K. Ronald, A. D. R. Phelps, and A. W. Cross, "Generation of broadband terahertz radiation using a backward wave oscillator and pseudospark-sourced electron beam," Appl. Phys. Lett., 107, no. 13, pp. 133501-1-133501-4, Sep. 2015.

[4] W. He, C. R. Donaldson, L. Zhang, K. Ronald, P. McElhinney, and A.W. Cross, "High power wideband gyrotron backward wave oscillator operating towards the terahertz region," Phys. Rev. Lett., vol. 110, no. 16, pp. 165101-1-165101-5, Apr. 2013.

[5] J. X. Wang, G. X. Shu, G. Liu, L. Y. Yang, and Y. Luo, "Ultrawideband C oalesced-mode operation for a SheetBeam Traveling-Wave Tube," IEEE Trans. Electron Devices, vol. 63, no. 1, pp. 504-511, Jan. 2016.

[6] G. Liu, J. X. Wang, G. X. Shu, Y. Luo, L. Y. Yang, and S. Wang, "RF Design, thermal analysis, and cold test of a $\mathrm{Ku}-$ Band continuous wave sheet beam traveling wave tube," IEEE Trans. Electron Devices, vol. 62, no. 11, pp. 3844-3850, Nov. 2015.

[7] A. Baig, Y. M. Shin, L. R. Barnett, et al., "Design, fabrication and RF testing of near- $\mathrm{THz}$ sheet beam TWTA," IEEE Trans. on Terahertz Science and Technology, vol.4, no.4, pp. 181-207, Apr. 2011.

[8] A. W. Cross, H. Yin, W. He, et al., "Generation and application of pseudospark-sourced electron beams," $J$. Phys. D: Appl. Phys., vol. 40, no. 7, pp. 1953-1956, Mar. 2007.

[9] H. Yin, A. W. Cross, W. He, et al., "Millimeter wave generation from a pseudospark-sourced electron beam," Phys. Plasmas, vol. 16, no. 6, pp. 063105-1-063105-6, Jun. 2009. 
[10]H. Yin, A.W. Cross, A. D. R. Phelps, et al., "Propagation and post-acceleration of a pseudospark-sourced electron beam," Appl. Phys. Lett., vol. 91, no.8, pp. 5419-5422, Apr. 2002.

[11]H. Yin, G. R. M. Robb, W. He, et al. "Pseudospark-based electron beam and cherenkov maser experiments," Phys. Plasmas., vol. 7, no.12, pp. 5195-5205, Dec. 2000. 